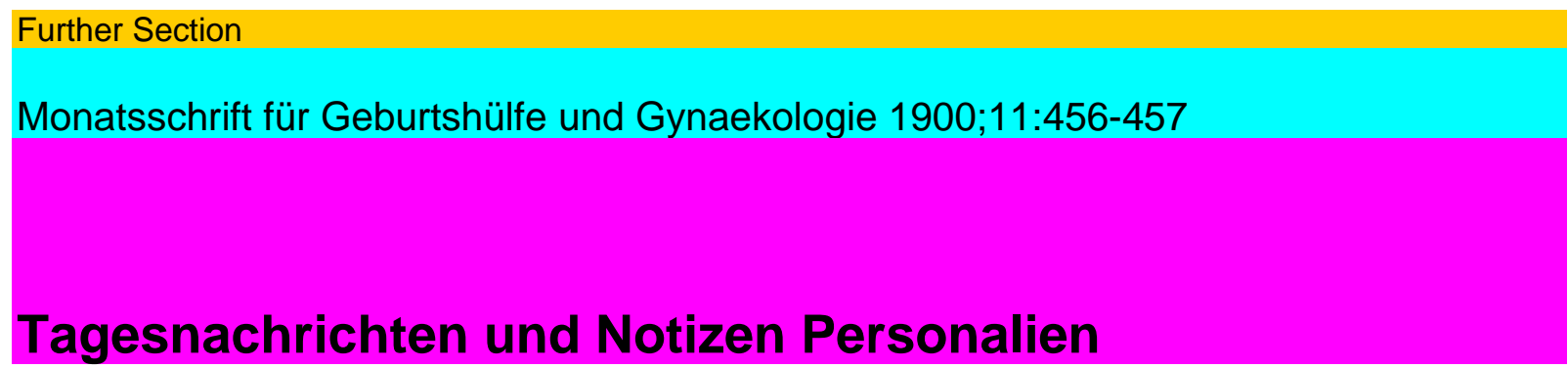

\title{
Friedpich Sehauta.
}

Im Verlaufe des Jahres 1899 hat Friedrich Sehauta das 5. Quinquennium seit seiner Promotion zurückgelegt. In der Schule von Späth lierangewachsen, hat Sehauta in engem Anschluss 3/4n die deutsche Gynäkologie, speziell die Berliner Schule, eine eigen-artige selbständige Entwickelung durchgemacht. Die Früchte dieser Entwickelung liegen in den Piiblikationen besonders aus der Zeit seiner Leitung der Kliniken in Innsbruck, Prag und

Geor $\cdot g$ Heinrich Peter Krukenberg $\uparrow 457 \mathrm{~s}$

Wien den Fachgenossen zutage. Er selbst hat dann in dem „Lel $\prod \cdot-b u c h$ der gesamten Gynäkologie" seiner Scliule ,eiøe Grundlage e·e ¿reben, auf welcher sich. díeselbe in überaus fruchtbarer Weise zu einer hochbedeutenden entwickelt hat. - Die Redaktion und der Verleger dieser Monatsschrift haben sick in voller Anerkennung der Bedeutung Sckautas und seiner Scliule gern dem $\lambda$ Vunsche von Scliauta's Sckülern dienstbar gemacht, um eine reiclie Sainmlung von Arbeiten aus Scliauta's Klinik zu einer Festschrift zu vereinigen. Dieselbe soil als ein Zeichen berzlicher Dankbarkeit darüber Rechenschaft geben, in wie ausgiebiger Weise es Schauta gelungen ist, seine Schüler zu eigener Beobachtung und eigener Arbeit anzuregen. Diese Festschrift spricht fur sich selbst. Wir aber wollen dem hocliverdienten Kollegen und lieben Freunde auch unsererseits unsere herzlichsten Glückwünsche zu diesem wissenschaftlichen Jubiläum darbringen. Mögen noch lange Lustren segensreicher Arbeit an die bislierigen sich anreihen, möge ihm selbst diese so fruchtbare Tliätigkeit als Forscher und Lehrer innige Genugthuung und Freude bereiten und ihn die schwere Arbeitslast als klinischer Lehrer, Forscher und Arzt in der $\mathrm{Zu}-$ versicht eines noch immer wachsenden, reichen Erfolges freudig tragen lassen. A. Martin. M. Sänger.

Georg $\cdot$ Heinrich Peter Krukenberg $\uparrow$.

Professor Krukenberg, geboren zu Calbe 1G. December 1855, stauimt aus einer Paniilie berühmter Mediciner. Sein Urgrossvater Reil, Professor der inoere $\alpha$ Medicin in Halle und Berlin, war der. oberste Letter aller preussischen Kriegslazarethe im Jahr 1813. Reil starb am 2’2. November 1813 in Halle am Typhus - ein Opfer seines Berufs. Dessen Tochter war die Gattin Peter Krukenberg's, des berübmtesten Klinikers seiner Zeit.

Krukenberg selbst genoss den Schulunterricht in Halle. Er stiidírte in Tubingen, Strassburg und Halle, wo er 1878 sein Staats- $€ x a m e n$ bestand. Nachdem er sich eine allgemeine mediciuisclie Aus-bildung als Assistenzarzt am herzo $3 / 8$ lichen Krankenhause in Braunschweig erworben hatte, wurde er unter Ye it, e'mem Jugendfreunde seines Vaters, Assistenzarzt an der „gynaekologischen Klinik” in Bonn. Sehon in jungen Jahren hatte Krukenberg eine vortreffliche Studie über $<$ len Flecktyphus geliefert. El $>$ euso bewegten sich seine ersten Bonner Arbeiten „über die Wirkung tem|)erirter Vollbäder” noch auf dem Gebiete der innereu $\mathrm{Me}<$ iicin. 
Paid nachdem Krukenberg in die Klinik zu Bonn eingetreten war, ftel ihm das gesammte wissenschaftliche Material zur Bearbeitung aiiheim. Die Bonner Frauenklinik verdankt seinem Fleisse viel. Ein Yortrett'licher Catalog mit wissenschaftliclien und literarischen Be-merkuugen ist noch heute ein Beweis seines vnermüdlichen Eifers.

Seine Arbeiten waren nicht einfache Berichte über alltägliche Ereignisse eines Krankenhauses, vielmehr hat sich Krukenberg an die grössten und scliwierigsten, zum Theíl auch heute noch nicht deftnitiv gelösteu Probleme der Wissenscliaft lierangewagt. Die wiclrtigsten Beiträge 